\title{
An examination of attentional control in the auditory modality: Further evidence for auditory orienting
}

\author{
PHILIP T. QUINLAN and PETER J. BAILEY \\ University of York, Heslington, York, England
}

\begin{abstract}
Four experiments are reported that examine attentional control in the auditory modality. In Experiment 1 , the subjects made detection responses to the onset of a monaurally presented pure tone that was preceded by a pure-tone cue. On a valid trial, the cue was presented in the same ear as the target; on an invalid trial, it was presented in the contralateral ear to the target; and on a neutral trial, it was presented in both ears. Overall performance was facilitated on valid trials in comparison with invalid trials. In later experiments, the subjects made choice decisions about the location of the target, and significant cuing effects were found relative to the neutral condition. Finally, performance was assessed in the presence of central (spoken) word cues. Here, the content of the cue specified the likely location of the target. Under these conditions, costs and benefits were found over a range of cue-target stimulus onset asynchronies. The results are discussed in terms of automatic and controlled attentional processes.
\end{abstract}

One of the most studied techniques for examining attentional control consists of visual cuing. In such experiments, an initial visual signal (the cue) precedes the presentation of the visual target to which the subjects respond. Performance is then examined as a function of the nature of the cue. Typically, three types of cue have been usednamely, valid cues, which correctly indicate the location of the target; neutral cues, which provide no information as to the location of the target; and invalid cues, which indicate a location different to that at which the target occurs. It has been found that reaction times (RTs) on valid trials are faster than those on neutral trials (i.e., that benefits accrue to validly cued targets), and, in contrast, that RTs on invalid trials are slower than those on neutral trials (i.e., that costs accrue to targets preceded by invalid cues). Through the analysis of such costs and benefits, much has been learned about visual attention, and about the cuing paradigm itself. More recently, though, interest in auditory attention has grown (see Scharf, 1988, for a review), and although there is considerable potential in exploring auditory attention with similar cuing techniques to those used with vision, many basic experiments have yet to be carried out.

Part of the reason for the lack of research into auditory attention may lie in the failures to show significant ef-

This work was supported by a grant from the University of York Innovation and Research Priming Fund. Thanks are due Greg Davis, who ran the experiments, and Jon Driver and Charles Spence, for allowing us access to pre-prints of their article. The research has benefited from discussion with Jon Driver and Hermann Müller. Correspondence regarding this paper should be addressed to P. T. Quinlan, Department of Psychology, University of York, Heslington, York YO1 5DD, England (e-mail: ptq1@york.ac.uk) fects of spatial uncertainty on auditory signal detection (e.g., Lowe, 1968). In addition, Posner (1978), in his extremely influential book, reported a failure to find any interesting cuing effects with auditory targets. He found neither costs nor benefits when simple tone targets were coupled with visual cues. Moreover, no cuing effects were observed either in simple target-detection tasks or in tone-discrimination tasks.

A more recent failure to find evidence of auditory cuing has been reported by Buchtel and Butter (1988). They used the different combinations of visual and auditory cues and targets to examine possible within- and between-modality cuing effects. Essentially, they found significant auditory cuing effects when subjects were cued with a burst of white noise and made detection responses to a light; however, when visual and auditory cues were used with auditory targets, no significant cuing effects were found at any of the tested stimulus onset asynchronies (SOAs).

Although such negative results seemingly demonstrate that there is no auditory equivalent of visual orienting (see Buchtel \& Butter, 1988; Butter, Buchtel, \& Santucci, 1989), several other papers have appeared that do report significant auditory cuing effects. For example, Murray, Allard, and Bryden (1988) presented subjects with binaural cues (i.e., the spoken words "left," "right," and "center") and the task was to respond to the location of the spoken sound [do], which occurred in either the left or the right ear. The results were clear-cut in showing significant benefits, but no costs, relative to the neutral ("center") trials. Using a slightly different method, Mazzucchi, Cattelani, and Umiltà (1983) also found significant cuing in a study involving simple RTs to monaural clicks. They reported a 5-6-msec cost when 
the target click was preceded by an invalid verbal auditory cue, and a 6-7-msec benefit when the click was preceded by a valid musical tone.

More recently, though, an extensive series of experiments, carried out by Spence and Driver (1994), has shown unequivocally that auditory orienting does take place. In their first experiment, Spence and Driver used an uninformative cue tone with white noise targets. Each subject sat between two rows of three speakers. One speaker in each row was situated in front of the subject, while the middle speaker in each row was aligned with the subject's ears and the remaining speaker was situated behind the subject, who made choice responses according to whether the target fell in front of or behind him or her; RTs were measured. Performance was assessed as a function both of the cue's validity and of the SOA between the cue and the target. In this case, on valid trials, the cue was presented in the same row of speakers as the target, and on invalid trials, the cue and the target were presented in opposite rows. Spence and Driver uncovered a statistically significant advantage for responses on valid trials, in comparison with invalid trials, when an SOA of $100 \mathrm{msec}$ was examined. No differences arose at longer SOAs. They discussed this pattern of results in terms of a short-lived, automatic orienting mechanism.

In a later experiment, Spence and Driver (1994) used informative cues with the same paradigm. This time, the cue predicted the side of the target on $75 \%$ of the trials. Under these conditions, significant cuing effects arose at all SOAs tested. Spence and Driver interpreted performance under these conditions in terms of the operation of both endogenous and exogenous shifts of attention. In essence, the endogenous/exogenous distinction maps onto the controlled/automatic distinction discussed in the visual-attention literature (see Folk, Remington, \& Johnston, 1992), where it has been argued that whereas exogenous factors operate at short SOAs, endogenous factors operate at longer SOAs (see, for example, Müller $\&$ Rabbitt, 1989). According to this view, whereas endogenous processes may reflect strategic modifications imposed by the subject, exogenous processes are immune to such modifications. Interestingly, Spence and Driver report that when performance with uninformative cues was compared with performance with informative cues, the cuing effects at the shortest SOA (i.e., at $100 \mathrm{msec}$ ) were enhanced in the latter case relative to the former-a result they interpreted as showing that the exogenous component can be modulated by task constraints. Indeed, Spence and Driver argued that their results showed that auditory orienting is not wholly automatic, because it can be modified by intention.

Since so few studies have explored auditory orienting in any detail, the present paper starts with an examination of possible cuing effects in the context of a simple target-detection task. In the present case, the auditory stimuli were delivered over headphones (instead of external speakers); hence, the location of the sound source was unambiguous. Such conditions provide the optimal context in which any form of auditory orienting might be found. In addition, a manipulation of cue probability was included. 1

The rationale for this manipulation derived from aspects of the visual-information-processing literature, most notably the work of Posner and Snyder (1978). In their first experiment, they presented subjects with a priming field comprising either a neutral plus sign or a capital letter, followed immediately by a target field comprising two capital letters. The subjects had to decide rapidly whether or not the letters were identical. When the target letters were identical, the prime either matched the identity of the target letters or it did not. Across different conditions, the probability of a primetarget match was manipulated: in the 80-20 condition, matching trials outnumbered mismatching trials; in the 20-80 condition, this proportion of trial types was reversed; and in the 50-50 condition, there were equal numbers of matching and mismatching trials. Posner and Snyder reported significant benefits on matching trials in all three conditions, with the size of the effects reflecting the proportion of matching to mismatching trials. Moreover, the size of the benefits was greater than the size of the costs in all three conditions.

In assessing these results, Posner and Snyder (1978) sketched how the data fitted with Keele's (1973) analysis of attention, the central idea of which concerned the prime automatically activating the internal representations and pathways relevant to the processing of the target letters. According to this view, at short prime-target intervals, only facilitation effects will be observed, because automatic activation of the targets' representation has no inhibitory consequences for any other part of the memory system. In contrast, at longer prime-target intervals, the subject's conscious attention to the prime will have deleterious consequences for the processing of subsequent information, because conscious attention is limited in capacity, and therefore attending to one stimulus inhibits attending to another. As a result, at short prime-target intervals, only benefits should occur, and at longer prime-target intervals, both benefits and costs should occur. Indeed, when Posner and Snyder systematically manipulated the interstimulus interval between prime and target, the pattern of results fitted the predicted pattern very well. Furthermore, this general framework was used to provide an explanation of performance when the cue-target probability was manipulated. It was argued that uninformative primes (used in the 50-50 condition) should produce only automatic activation, and hence significant benefits but no costs. In contrast, informative primes (used in the 80-20 condition) should produce both significant costs and benefits.

While the general pattern of results supported these notions, little consideration was given to performance in the 20-80 condition. Performance on these trials is of interest because any effects due to the automatic activation of the target letters' representation can be disentangled from other effects that derive from the manipulation of the probability of a particular trial type. In practice, the informativeness of the prime in the $80-20$ condition is 
confounded with the fact that a matching trial occurred more frequently than a mismatching trial. This was not the case in the 20-80 condition, in which the improbable event was a matching trial. Nevertheless, the data from the 20-80 condition revealed that the prime did facilitate performance when it matched both target letters, in comparison with when it did not, and that there were no costs associated with the prime on mismatching trials. In this respect, performance in the 20-80 condition fits well with the idea that automatic facilitation can occur in the absence of any associated costs. Clearly, cue type and probability of cue can produce differential effects, and what follows describes an attempt to examine these effects systematically in auditory perception.

The primary issue under investigation in the first experiment was whether auditory orienting could be established under conditions in which the location of the sound was unambiguous. A secondary issue was whether it could be affected by small differences in the probability of a given cue type. The later experiments examined the effects of manipulating cue-type probability in more detail. Importantly, though, in none of the experiments were any of the subjects informed about the probability of the various trial types and/or the manipulation of this probability; this was in order to examine the operation of attentional processes in conditions in which subjects could not systematically formulate expectations of particular trial types. Such conditions were thought ideal for tapping the effects of automatic processes.

\section{EXPERIMENT 1}

In the first experiment, we sought to measure auditory orienting in a simple tone-detection task. Cues and target signals were both pure tones, and subjects made detection responses to the onset of the target. All stimuli were delivered over headphones. The SOA in the experiment was set at the duration of the cue (i. e., $100 \mathrm{msec}$ ). Applying the logic of the Posner and Snyder (1978) study, the task should reflect the operation of automatic processes, and hence processing benefits should be expected on valid trials, but costs should not be expected on invalid trials. As a further test of this notion, a minor manipulation of the probability of the cue type was introduced to see whether any cuing effects would be modulated by the probability of a given cue type. If auditory orienting processes were purely automatic, no effect of a cue-type-probability manipulation should be expected.

\section{Method}

\section{Subjects}

Twelve undergraduates from the University of York acted as subjects. Some received course credit for participating, while others were paid $£ 3$. They were selected without regard to gender, and no subject reported a hearing impairment.

\section{Stimuli and Apparatus}

Both cue and target were pure tones lasting $100 \mathrm{msec}$, including 5 -msec linear ramps in the amplitude envelope at onset and offset.
The frequencies of the cue and target tones were $2000 \mathrm{~Hz}$ and $400 \mathrm{~Hz}$, respectively. The tones were synthesized digitally with a sampling rate of $10 \mathrm{kHz}$, and were presented via 12-bit digital-toanalog converters, postprocessing filters (low pass, $100 \mathrm{~dB} /$ octave, $3.5-\mathrm{kHz}$ corner frequency), digitally controlled attenuators, and Sennheiser HD414 headphones. Stimulus intensities were set to give peak sound pressure levels of $75 \mathrm{~dB}(\mathrm{~A})$ at the headphones, calibrated with a Bruel and Kjaer artificial ear and sound level meter. Stimulus timings, presentation, and data collection were controlled by computer.

Each subject was tested individually in a sound-attenuating chamber in which the ambient noise level was less than $40 \mathrm{~dB}$ (SPL). The subject sat at a table and wore headphones throughout the experiment. On the table was a response box incorporating a number of finger keys. The subject responded by pressing a single response key with the index finger of his or her preferred hand.

\section{Design}

There were four trial types: valid, invalid, neutral, and catch. On valid trials, cue and target had location in common, so the cue was presented to either the left or the right ear, and was followed immediately by the target in the same ear. On invalid trials, cue and target were at different locations, so when the cue was presented to the left ear, it was followed immediately by the target in the right ear, or vice versa. On neutral trials, the cue was presented simultaneously to both ears, and so was perceived in a central location, followed immediately by the target in either the left or the right ear. When the cue was presented binaurally, its intensity was decreased by $3 \mathrm{~dB}$, and its loudness was approximately equal to that of the cue when presented monaurally. On catch trials, the cue alone was presented (i.e., no target followed its presentation). Catch trials included left, right, and central cues with equal frequency.

All subjects served in each of the three conditions, which differed in terms of the relative proportions of the four different trial types: In the $80 \%$ set, there were 128 valid trials, 32 invalid trials, 80 neutral trials, and 48 catch trials, and in this case, $80 \%$ of the trials were cuing trials; in the $83 \%$ set of experimental trials, there were 160 valid trials, 32 invalid trials, 48 neutral trials, and 48 catch trials, and in this case, $83 \%$ of the trials were cuing trials; and in the $86 \%$ set of experimental trials, there were 192 valid trials, 32 invalid trials, 16 neutral trials, and 48 catch trials; in this case, $86 \%$ of the trials were cuing trials. The order in which the conditions were presented was counterbalanced across subjects.

\section{Procedure}

At the start of each testing session, the subjects were informed about the nature of the task and were told that typically on each trial, a high-pitch tone (the cue) would precede a low-pitch tone (the target). They were told to respond as quickly as possible when they heard the low-pitch target tone, and they were also informed that on some trials, no target would be presented, and that on these trials, no key response should be made. Such catch trials were included to inhibit the subjects from simply pressing the key in anticipation of the target. The subjects were given no further information about the cue-target probabilities.

Each response initiated an intertrial interval that had a mean duration of $1,200 \mathrm{msec}$, but that varied randomly from trial to trial within the range $1,000-1,400 \mathrm{msec}$. For catch trials on which no response was made, the intertrial interval was initiated $700 \mathrm{msec}$ after the offset of the target tone.

The subjects were given an opportunity to practice the task in each of the three experimental conditions. A total of 36 practice trials were given in each condition. Following the initial practice block, the subjects were asked whether they had experienced any difficulty in hearing the stimuli and whether they were able to distinguish the cue and target tones. None of the subjects reported any 
such difficulties. After the practice trials, each condition was presented as four blocks of 72 trials, with brief rest periods between each block.

\section{Results}

Summary statistics of the conditions of interest are shown in Table 1. All RTs that were less than $100 \mathrm{msec}$ or further than $\pm 3 S D$ s away from each subject's condition mean were discarded from the analyses. This served to minimize the effect on the RT data of the very short latencies associated with anticipations, which, if included in the analysis, would weaken the argument that performance reflects the operation of attentional processes rather than, say, strategic effects (Duncan, 1980). Similarly, overlong responses should tend to skew the data in the opposite direction, and hence they, too, were eliminated. The corresponding percentages of outliers are presented in Table 1.

The RT data were entered into a $3 \times 3$ repeated measures analysis of variance (ANOVA), with condition $(80 \%, 83 \%$, and $86 \%)$ and cue type (valid, invalid, and neutral) as fixed factors and subjects as a random factor. The analysis revealed only a statistically significant main effect of cue type $[F(2,22)=6.19, p<.01]$. Neither the main effect of condition nor the condition $X$ cue type interaction was statistically reliable (both $F_{\mathrm{s}}<1.0$ ).

Further exploration of the main effect of cue type revealed that the only statistically significant pairwise difference was that between the data for the valid and the invalid trials ( $p<.01$, Tukey HSD test). The mean RTs for the valid, invalid, and neutral trials were $261 \mathrm{msec}$, $270 \mathrm{msec}$, and $265 \mathrm{msec}$, respectively. The post hoc test revealed that the $9-\mathrm{msec}$ difference between the data for the valid and the invalid trials was statistically reliable.

The percentages of errors committed on the catch trials in the $80 \%, 83 \%$, and $86 \%$ conditions were $10.76 \%$, $15.45 \%$, and $15.45 \%$, respectively. Error rates for each subject were computed and analyzed in a one-way repeated measures ANOVA, with condition as a fixed factor and subjects as a random factor. Errors were arcsin transformed (Winer, 1962) prior to being analyzed. The analysis revealed a statistically significant main effect of condition $[F(2,22)=3.61, p<.05]$. Although post hoc tests failed to reveal the statistical reason for this result, numerically fewer keypresses were made on the catch trials in the $80 \%$ condition than in the other two condi- tions. There is no obvious reason for this result, and it appears not to have systematically affected performance in the three conditions on trials during which the target was presented.

\section{Discussion}

The present experiment has revealed a small but statistically reliable difference between responses on valid and invalid trials. Inspection of the data revealed that 11 of the 12 subjects responded faster to valid trials than to invalid trials. The experiment has demonstrated that auditory orienting does take place even when a simple detection response is required. However, the small size of the effect perhaps accounts for why others have failed to find comparable results, notably under conditions in which the stimuli were not presented via headphones. Nevertheless, the size of these effects is directly comparable to that reported by Mazzucchi et al. (1983) when they measured simple RTs to monaural clicks. The more specific predictions concerning the relative size of the costs and benefits cannot be addressed, simply because the differences relative to the neutral baseline were not statistically significant. Given that the obtained cuing effect was so small, the absence of any additional effect of the limited manipulation of cue-type probability is not surprising. The effects of systematic variation in the probability of cue type were explored in Experiment 2, using a different cuing methodology.

\section{EXPERIMENT 2}

Although Experiment 1 has revealed that auditory cuing can occur when subjects produce simple RTs to the onset of a pure tone, the effects are small and therefore difficult to examine in detail. As a consequence, an alternative methodology was adopted in which subjects were required to make choice RTs. In this case, the subjects were provided with left and right response keys and were required to press the key corresponding to the location of the target. When the target arrived at the left ear, the subjects had to press the left key; when the target arrived at the right ear, they had to press the right key. A similar response arrangement has been used repeatedly in experiments of visual cuing (see Maylor, 1985, Experiment 1c; Posner, Nissen, \& Klein, 1976; Posner, Nissen, \& Ogden, 1978; Simon, Acosta, \& Mewaldt,

Table 1

Mean Reaction Times (in Milliseconds) and Percentages of Outliers* for Experiment 1

\begin{tabular}{|c|c|c|c|c|c|c|c|c|}
\hline \multirow[b]{3}{*}{ Condition } & \multicolumn{6}{|c|}{ Cue Type } & & \\
\hline & \multicolumn{2}{|r|}{ Valid } & \multicolumn{2}{|r|}{ Neutral } & \multicolumn{2}{|r|}{ Invalid } & \multicolumn{2}{|c|}{ Effect } \\
\hline & $\overline{\mathrm{RT}}$ & $\overline{\text { Outliers }(\%)}$ & RT & Outliers (\%) & RT & Outliers (\%) & Benefit ${ }^{\dagger}$ & $\overline{\text { Cost }}$ \\
\hline $80 \%$ valid & 258 & 3.6 & 260 & 4.2 & 267 & 2.6 & 2 & 7 \\
\hline $83 \%$ valid & 261 & 2.4 & 264 & 3.3 & 267 & 3.6 & 3 & 3 \\
\hline $86 \%$ valid & 264 & 2.4 & 273 & 2.9 & 276 & 3.1 & 9 & 3 \\
\hline
\end{tabular}

*See text for explanation of percentages of outliers. "The benefits were computed by subtracting the valid scores from the neutral scores. †The costs were computed by subtracting the neutral scores from the invalid scores. 
1975). When such a methodology is used, however, performance may reflect the operation of either or both of two sets of processes. Specifically, these are perceptual/ attentional mechanisms and response preparation (see Posner et al., 1978). For instance, any projected facilitation of performance might reflect some form of enhancement of the stimulus input due to the application of selective attention, and inhibition of performance might reflect poor stimulus encoding due to attention being directed elsewhere. Alternatively (or additionally), the projected costs and benefits may reflect differential response preparedness caused by the presentation of the cue prior to the target. Both possible accounts are explored in detail below, and a later experiment addresses the issue directly. Initially, however, our primary interest was with the possible effects that might arise when cue probability was systematically manipulated. The subjects were presented with the cue-target pairings as before, but they now made responses on the basis of the location of the target - that is, they pressed the right key when the target fell in the right ear, and the left key when it fell in the left ear. Across different blocks of experimental trials, there were three main conditions: a 20-80 condition ( $20 \%$ valid trials, $80 \%$ invalid trials), a $50-50$ condition, and a $80-20$ condition. The $20-80$ condition was of particular interest because in this condition, presentation of the valid cue was the least probable event, thus maximizing the likelihood that any benefits associated with the cue reflected the operation of automatic processes.

Apart from the original Posner and Snyder (1978) paper, very few studies have manipulated cue-type probability in a manner similar to that used here. One such case, though, is reported by Jonides (1980), in which subjects made choice RTs to displays of letter stimuli arranged in a circle around a central fixation point. They made a right keypress if the letter $R$ was present and a left keypress if the letter $L$ was present. Prior to the presentation of the letters, a centrally positioned arrowhead symbol (the cue) was presented, and it pointed to one of the subsequent letter positions. On neutral trials, the cue was a centrally positioned diamond symbol. Across different blocks of trials, Jonides varied the probability with which the cue accurately predicted the location of a possible target letter, so that it was valid on either $70 \%$, $50 \%$, or $30 \%$ of the cuing trials. The results showed that the size of the cuing effects varied systematically as a function of cue-type probability. When the cue was informative about the actual location of the target, significant costs and benefits were found. Costs and benefits (the latter only marginally significant) arose when the cue was uninformative. No significant cuing effects arose when the valid cue occurred infrequently. Overall, this pattern of results fits rather uneasily with the twocomponent account of attention discussed by Posner and Snyder, according to which the automatic component ought to operate on all valid trials, regardless of the probability of a valid cue, with the result that equivalent benefits should be predicted for all three conditions.
Consequently, the finding that the benefits decreased as a direct function of the probability of a valid cue stands in contrast to the two-component account. For this reason, Jonides was forced to consider other plausible explanations of performance, one of which forms the basis of the later discussion of the current results.

Before describing the present experiment, it is important to note a critical difference between the procedure Jonides (1980) adopted and that adopted here. Prior to each experimental block of trials, Jonides informed his subjects what the exact proportion of the different types of trials would be. In this respect, the subjects were encouraged to build up expectations of the composition of the blocks of trials as the trials progressed. In the present study, however, the subjects were given no such instructions, and thus began each block ignorant of the block composition. The present experiment therefore provides better conditions under which automatic processes may be examined.

\section{Stimulus and Apparatus}

\section{Method}

The experiment was run in the same laboratory as Experiment 1 , with the same equipment and stimuli.

\section{Subjects}

Eighteen undergraduates from the University of York acted as subjects. Some accrued course credit for participating, while others were paid $\mathfrak{k} 3$. As with the previous experiment, the subjects were selected without regard to gender, and none reported a hearing impairment.

\section{Design}

The experiment was organized around a repeated measure design: each subject ran through three different conditions in a single session. In each of the conditions, there were three trial types-valid, invalid, and neutral, as defined previously.

The three conditions differed in terms of the proportion of valid and invalid trials. In the $80-20$ condition, there were 128 valid trials, 32 invalid trials, and 80 neutral trials; of the valid and invalid trials, $80 \%$ were valid. In the $50-50$ condition, there were 80 valid trials, 80 invalid trials, and 80 neutral trials; of the valid and invalid trials, $50 \%$ were valid. Finally, in the $20-80$ condition, there were 32 valid trials, 128 invalid trials, and 80 neutral trials; of the valid and invalid trials, $20 \%$ were valid.

\section{Procedure}

The procedural details were identical in many respects to those described for Experiment 1; the critical difference, however, concerned the response requirements. The subjects now rested both hands on the response box and were told to press the finger key that was the farthest left with their left index finger and to press the finger key that was the farthest right with their right index finger. The experiment required that they press the left key whenever the target was presented to the left ear and the right key whenever the target was presented to the right ear.

For each of the three conditions, 4 consecutive blocks of 60 trials were presented. Each condition had an associated block of initial 30 practice trials. The order of presentation of conditions was counterbalanced across the subjects.

The subjects were informed about the nature of the task and were told that on each trial, a high-pitch tone (the cue) would precede a low-pitch tone (the target). They were given no additional information about the manipulation of cue-type probability across the different blocks of experimental trials. They were told to re- 
spond as quickly as possible when they heard the low-pitch target tone and to press the finger key on the side on which the target tone was presented. However, they were also told not to sacrifice accuracy for speed.

\section{Results and Discussion}

All RTs of less than 100 msec or that were more than $\pm 3 S D$ s away from each subject's condition mean were discarded from the analysis. RTs and response errors were analyzed in a corresponding manner. Errors were expressed as percentages and arcsin transformed (Winer, 1962) prior to analysis. Summary statistics of the RT data are shown in Table 2.

\section{RTs}

The RT data were entered into a $3 \times 3$ repeated measures ANOVA, with condition (80-20, 50-50, or 20-80) and cue type (valid, invalid, or neutral) as fixed factors and subjects as a random factor. The analysis revealed a statistically significant main effect of cue type $[F(2,34)=$ $99.78, p<.001]$ and a statistically reliable condition $\times$ cue type interaction $[F(4,68)=6.46, p<.001]$. The main effect of condition failed to reach statistical significance $[F(2,34)=1.11, p>.05]$.

In order to examine the statistically significant interaction in more detail, the size of the costs and benefits relative to the neutral trials was computed for each condition. The resulting difference scores were entered into a $3 \times 2$ repeated measures ANOVA, with condition (80-20, 5050 , or 20-80) and cuing effects (costs or benefits) as fixed factors and subjects as a random factor. This new analysis revealed that only the main effect of condition reached statistical significance $[F(2,34)=8.70, p<.001]$. Neither the main effect of cuing effects nor the condition $\times$ cuingeffects interaction was statistically significant $(F<1.0$ for both). The overall pattern of results demonstrates that for each condition, the size of benefits was equivalent to the size of costs. The statistically significant main effect of condition shows, however, that across the three conditions, the overall magnitude of the cuing effect varied. A further exploration of the significant main effect of condition revealed that overall, the cuing effects were smaller in the 20-80 condition than they were in either of the other two conditions ( $p<.05$ for both, Tukey HSD test). The size of cuing effects in the 50-50 and 80-20 conditions did not differ $(p>.05$, Tukey HSD test). Overall, the mean cuing effects in the $20-80,50-50$, and 80-20 conditions were, respectively, 36,50 , and $58 \mathrm{msec}$.

Table 2

Mean Reaction Times (in Milliseconds) for Experiment 2

\begin{tabular}{|c|c|c|c|c|c|}
\hline \multirow[b]{2}{*}{ Condition } & \multicolumn{3}{|c|}{ Cue Type } & \multicolumn{2}{|c|}{ Effect } \\
\hline & Valid & Neutral & Invalid & Benefit* & Cost $†$ \\
\hline $20-80$ & 341 & 373 & 413 & 32 & 40 \\
\hline $50-50$ & 339 & 385 & 438 & 46 & 53 \\
\hline $80-20$ & 315 & 371 & 430 & 56 & 59 \\
\hline
\end{tabular}

* The benefits were computed by subtracting the valid scores from the neutral scores. 'The costs were computed by subtracting the neutral scores from the invalid scores.
The overall pattern of RTs has shown symmetrical costs and benefits in all three conditions. Whereas costs and benefits were equivalent in the 50-50 and 80-20 conditions, smaller effects were found in the $20-80$ condition. Nevertheless, both significant costs and significant benefits did arise in the $20-80$ condition. The present RT data stand in contrast to those reported by Posner and Snyder (1978) and by Jonides (1980). Posner and Snyder found only benefits and no costs in their 20-80 condition. They took this to reflect the operation of a purely automatic component associated with pathway activation of the common processing route for both the cue and target letters. Jonides, in contrast, found neither costs nor benefits when the valid cue was presented on only $30 \%$ of cuing trials. In the present experiment, yet another pattern was observed: both costs and benefits were found when the valid cue was presented on only $20 \%$ of cuing trials. Moreover, these effects arose at a short SOA, and so might be associated with the operation of an automatic component. Nevertheless, the presence of RT costs at such a short SOA does not fit comfortably with Posner and Snyder's simple two-component model. It is appropriate to consider, therefore, the extent to which these effects are associated with the operation of a purely automatic component. A more lengthy discussion of these points is deferred until later.

\section{Errors}

Table 3 summarizes the error data for this experiment. The arcsin-transformed errors were examined in the same sort of ANOVA as was used for the RT data. In this case, the main effect of condition $[F(2,34)=3.52, p<$ $.05]$, the main effect of cue type $[F(2,34)=31.11, p<$ $.001]$, and the condition $\times$ cue-type interaction $[F(4,68)=$ $19.18, p<.0001]$ were all statistically reliable.

To examine this pattern of results in more detail, measures of costs and benefits were computed from the accuracy data. Differences from the neutral baselines were computed for the transformed error scores, and these data were entered into a $3 \times 2$ repeated measures ANOVA. Main effects of both condition $[F(2,34)=27.24, p<$ $.0001]$ and cuing effects $[F(1,17)=37.94, p<.001]$ were statistically reliable, as was the condition $\times$ cuing effects interaction $[F(2,34)=4.19, p<.05]$. Overall, the statistically significant main effect of condition showed that the smallest cuing effects arose in the $20-80$ condition, that larger effects arose in the 50-50 condition, and that the largest effects arose in the 80-20 condition ( $p<$ .05 for all comparisons, Tukey HSD test). The reliable main effect of cuing effects revealed that relatively more errors were made on invalid trials than on valid trials. However, the statistically significant condition $\times$ cuingeffects interaction showed that a particularly large number of errors were committed on the invalid trials in the 80-20 condition.

The finding that, across all three conditions, more errors were made on invalid trials than on valid trials reveals a tendency for subjects to respond with the key corresponding to the location of the cue. In order to ex- 
Table 3

Mean Percentages of Error Scores* for Experiment 2

\begin{tabular}{ccccc}
\hline & \multicolumn{3}{c}{ Cue Type } & \\
\cline { 2 - 4 } Condition & Valid & Neut ral & Invalid & Cued-Side Bias $\dagger$ \\
\hline $20-80$ & 1.04 & 1.31 & 5.38 & 0.76 \\
$50-50$ & 0.35 & 1.80 & 9.65 & 0.85 \\
$80-20$ & 0.47 & 1.32 & 14.76 & 0.84 \\
\hline
\end{tabular}

*These values were computed from only the incorrect responses; they do not include the outliers discarded prior to the RT analysis. +The derivation of the cued-side-bias values is given in the text.

amine the strength of any such response-bias effects in more detail, estimates of the cued-side bias were computed for each of the three conditions (see Table 3). Following Müller (1994), the cued-side bias is defined as the probability of guessing the cued side, given that guessing is taking place. Specifically:

$$
\text { Cued-side bias }=\frac{p(\mathrm{CC})}{p(\mathrm{CC})+p(\mathrm{IU})},
$$

where $p(\mathrm{CC})$ stands for the probability of committing a correct cued-side guess on valid trials, and $p$ (IU) stands for the probability of committing an uncued-side guess on valid trials. $p$ (IU) is simply the frequency of uncuedside errors committed on valid trials. $p(\mathrm{CC})$ can be estimated from the number of cued-side errors on invalid trials, but since there can be unequal numbers of valid and invalid trials, the cued-side error rate must be scaled in accordance with the proportion of valid to invalid trials. Hence, $p(\mathrm{CC})$ is given by

$$
\frac{\text { No. of valid trials }}{\text { No. of invalid trials }} \times \begin{gathered}
\text { No. of cued-side errors } \\
\text { on invalid trials }
\end{gathered}
$$

Estimates of cued-side bias greater than 0.5 reveal a tendency to respond to the location of the cue; in all three conditions, the estimates of cued-side bias were greater than 0.5 (see Table 3). To examine these estimates in more detail, values of the cued-side bias for each subject were computed and arcsin transformed prior to analysis in a one-way repeated measures ANOVA, with condition as a fixed factor and subjects as a random factor. This analysis showed that the main effect of condition failed to reach statistical significance $(F<1.0)$. Thus it appears that although subjects did exhibit a tendency to press the key associated with the location of the cue, this response bias did not vary across the three conditions. This indicates that the changes in the costs and benefits in the RT data across the three conditions cannot be attributed reliably to concomitant changes in response bias. Overall, therefore, the data not only show response bias, they show that this bias is in accordance with the finding that RTs on valid trials are quicker than RTs on invalid trials. However, the supplementary analysis shows that these response biases cannot provide a complete account of the data. Our claim is that the changes in the size of the cuing effects reflect the operation of an attentional component that is modulated by cue-target contingencies.

\section{EXPERIMENT 3}

Regardless of the further analyses concerning cuedside bias, the results of Experiment 2 still leave open the question about the degree to which the costs and benefits reported in the RT data may be attributed to attentional factors or to specific motor tendencies arising from priming of the particular response by the cue (see Posner et al., 1978). The measures of cued-side bias computed for the error data in Experiment 2 estimated the probability of guessing the cued-side location of the target, given that guessing was taking place. However, response bias may involve at least two potentially separable components. Guessing implies (possibly random) influences on response choice, whereas response priming implies influences on the ease with which a response can be made, and these influences may be relatively independent of each other. In Experiment 2, subjects exhibited a tendency to respond to the location of the cue, but this tendency did not account for the systematic changes in the cuing effects observed across the three conditions. Nevertheless, the cuing effects may have included components that were due to response priming, because subjects responded with the arm and finger on the side of the body corresponding to the target location. To explore these possibilities further, Experiment 3 again used a choice-RT paradigm, but the subjects were no longer required to respond with the limb associated with the side of the target; instead, they responded with the index and middle finger of their preferred hand (see Klein \& Hansen, 1990). If the target was presented to the right ear, they pressed the middle-finger key on the response box, and if the target was presented to the left ear, they pressed the index-finger key. Moreover, the response box was aligned so as to be perpendicular to the body, in order to minimize stimulus-position/fingerposition compatibility.

\section{Method}

\section{Subjects}

Twelve undergraduates from the University of York acted as subjects. Some accrued course credit for participating, while others were paid $£ 3$. As before, they were selected without regard to gender, and no subject reported a hearing impairment.

\section{Stimuli, Apparatus, and Procedure}

In all respects other than those described above, the experiment replicated Experiment 2.

\section{Results}

Summary statistics of the mean RTs for the conditions of interest are shown in Table 4. All RTs that were less than $100 \mathrm{msec}$ or further than $\pm 3 S D$ s away from each subject's condition mean were discarded from the analysis. Separate analyses were carried out on the RT and the error data. As before, the error data were collated from only the incorrect responses, and did not include the discarded RT outliers. 
RTs

Analysis revealed a statistically significant main effect of cue type $[F(2,22)=17.58, p<.0001]$ and a statistically reliable condition $\times$ cue-type interaction $[F(4,44)=$ $6.46, p<.0001]$. The main effect of condition failed to reach statistical significance $(F<1.0)$.

In order to examine the interaction in more detail, the size of the costs and benefits was analyzed further. This analysis revealed that only the main effect of condition reached statistical significance $[F(2,22)=13.37, p<$ $.001]$. Neither the main effect of cuing effects nor the condition $\times$ cuing-effect interaction were statistically significant $(F<1.0$ for both). The overall pattern of results demonstrates that for each condition the benefits and costs did not differ in size. The statistically significant main effect of condition shows, however, that across the three conditions, the overall size of the cuing effects varied. A further exploration of the significant main effect of condition revealed that overall, the size of the cuing effect was smaller in the $20-80$ condition than it was in either of the other two cases $(p<.05$ for both, Tukey HSD test). The size of cuing effect in the 50-50 and 80-20 conditions did not differ $(p>.05$, Tukey HSD test). Overall, the mean cuing effects in the 20-80, 50-50, and 80-20 conditions were, respectively, 24,47 , and $63 \mathrm{msec}$.

\section{Errors}

Table 5 shows summaries of the error data for this experiment. The arcsin-transformed errors were examined in the same type of ANOVA as was used with the RT data. As for the RT data, the main effect of condition failed to reach statistical significance $(F<1.0)$, while the main effects of cue type and the condition $\times$ cue-type interaction were statistically reliable $[F(2,22)=18.86$, $p<.0001$ and $F(4,44)=5.52, p<.05$, respectively].

To examine this pattern of results in more detail, measures of costs and benefits for the accuracy data were computed and analyzed accordingly. Only the main effect of condition reached statistical significance $[F(2,22)=10.29, p<.001]$. Neither the main effect of cuing effects $[F(1,11)=2.09, p>.05]$ nor the condition $\times$ cuing-effect interaction $[F(2,22)=1.68, p>.05]$ was significant. The statistically significant main effect of condition showed that cue type produced larger effects in the 80-20 condition than it did in the $20-80$ condition $(p<.01$, Tukey HSD test). No other comparisons reached statistical significance.

Table 4

Mean Reaction Times (in Milliseconds) for Experiment 3

\begin{tabular}{ccccccc}
\hline & \multicolumn{3}{c}{ Cue Type } & & \multicolumn{2}{c}{ Effect } \\
\cline { 2 - 4 } \cline { 5 - 6 } Condition & Valid & Neutral & Invalid & & Benefit* & Cost $\dagger$ \\
\hline $20-80$ & 413 & 454 & 462 & 41 & 8 \\
$50-50$ & 387 & 442 & 482 & 55 & 40 \\
$80-20$ & 374 & 433 & 490 & 59 & 57 \\
\hline
\end{tabular}

*The benefits were computed by subtracting the valid scores from the neutral scores. TThe costs were computed by subtracting the neutral scores from the invalid scores.
Table 5

Mean Percentages of Error Scores* for Experiment 3

\begin{tabular}{ccccc}
\hline & \multicolumn{3}{c}{ Cue Type } & \\
\cline { 2 - 4 } Condition & Valid & Neutral & Invalid & Cued-Side Bias $\dagger$ \\
\hline $20-80$ & 1.30 & 2.60 & 4.30 & 0.78 \\
$50-50$ & 0.94 & 3.65 & 5.63 & 0.66 \\
$80-20$ & 0.59 & 1.98 & 7.81 & 0.68 \\
\hline
\end{tabular}

*These values were computed from only the incorrect responses; they do not include the outliers discarded prior to the RT analysis. tThe derivation of the cued-side-bias values is given in the text.

In addition, estimates of cued-side bias were computed for each subject and were analyzed in the same way as in Experiment 2. Here again, the main effect of condition failed to reach statistical significance $(F<$ $1.0)$. Although the subjects did exhibit a tendency to press the key associated with the location of the cue, this response bias did not vary across the three different conditions, indicating that the changes in the costs and benefits in the RT data across the three conditions cannot be attributed to concomitant changes in response bias.

\section{Discussion}

Overall, there is good agreement between the results of Experiments 2 and 3. In both cases, the size of the costs and benefits in the RT data were symmetrical about the neutral baseline. Importantly, though, the overall size of the cuing effects was smaller in the 20-80 condition than it was in either of the other two conditions. In addition, significant benefits and costs were observed in the 50-50 condition when the cue was uninformative. This pattern of results does not fit well with Posner and Snyder's (1978) prediction that uninformative cues should produce benefits but not costs. Finally, significant benefits and costs were found in the 20-80 condition. It is therefore appropriate to consider the degree to which the auditory cuing effects can be explained in terms of the two-component model.

A useful recent treatment of this topic has been set out by Spence and Driver (1994). In their study of peripheral auditory cuing, they manipulated cue-type probability across experiments, and found that when the cue was informative, there were significant cuing effects at all SOAs tested. However, when the cue predicted the contralateral location of the target, significant cuing effects arose only at SOAs that were greater than $100 \mathrm{msec}$. This pattern of results is of interest because, like the results reported here, it does not fit well with traditional ideas about automatic processes, whereby, on the assumption that automatic processes cannot be interrupted and are insulated from strategic control, it would be predicted that, regardless of the cue probability, the size of the cuing benefit should have remained constant. However, Spence and Driver argued that subjects inhibit the exogenous orienting response when they know it will be detrimental to performance on most trials. This inhibition was evident at the shortest SOA, and hence it appears that the cuing effects found by Spence and Driver 
do not reflect the operation of an automatic process as such a process has traditionally been defined.

An important difference between the present study and the Spence and Driver (1991) study is that whereas in the latter, the subjects were fully informed of the cuetype probability, here, the subjects were given no such information. The difference between the two sets of results may therefore be attributable to whether or not subjects knew about the cue-type probability. When the subjects were ignorant of the cue-type probability, significant cuing effects arose at SOAs of $100 \mathrm{msec}$ when a valid cue occurred infrequently. In contrast, Spence and Driver showed that such cuing effects can be further modulated by subjects' expectations.

In all three conditions in both Experiments 2 and 3, the subjects showed a cued-side bias, with the average bias value being smaller in Experiment 3 than that in Experiment 2; in neither experiment, however, did this bias alter systematically as a function of the cue-type probability. The presence of the bias cannot therefore account for the variation in the size of the cuing effects across the different probability conditions. In Experiment 3, the subjects made responses with fingers of the same hand, and the response box was arranged so as to be perpendicular to the body midline. Thus, significant cuing effects were obtained even in the absence of a response procedure involving strong stimulus-response compatibility. This pattern of results strengthens the argument that the cuing effects reflect an attentional component, and provides further evidence for auditory attentional orienting.

Before turning to a discussion of auditory central cuing, it is important to consider a final alternative account of performance discussed originally by Jonides (1980). It is important because this account provides quantitative predictions about performance in the present tasks. The central idea is that attentional resources are allocated to possible target locations as a monotonic function of the likelihood that these locations will contain a target. This function is taken to reflect either the amount of attentional resources allocated to the various target locations (Eriksen \& Yeh, 1985) or the order in which the various target locations are accessed (Jonides, 1980). According to the latter account, and when the cue is less than $100 \%$ valid, the cued location is accessed first, but on only a proportion of trials. Variation in performance in different experimental conditions is explained with recourse to a procedure for internal probability matching, whereby subjects are assumed to attempt to adjust the probability of examining the cued location first in accordance with the overall probability of the cue validity. More recently, van der Heijden (1989) has provided a mathematical treatment of these ideas, the central assumptions of which are captured in Equations 3 and 4 below:

$$
\begin{aligned}
& \mathrm{RT}_{\text {valid }}=p_{\text {cue }}\left(\mathrm{RT}_{+}\right)+\left(1-p_{\text {cue }}\right) \mathrm{RT}_{\mathrm{n}}, \\
& \mathrm{RT}_{\text {invalid }}=p_{\text {cue }}\left(\mathrm{RT}_{-}\right)+\left(1-p_{\text {cue }}\right) \mathrm{RT}_{\mathrm{n}},
\end{aligned}
$$

where $R T_{\text {valid }}$ is the mean $R T$ on valid trials, $R T_{\text {invalid }}$ is the mean RT on invalid trials, and $p_{\text {cue }}$ is the probability of a valid cue.

By this account, the mean RT on a particular type of cuing trial is the sum of two components corresponding to the operation of focused and distributed attentional processes, respectively. ${ }^{2}$ The focused component reflects, in part, the amount of time taken to identify the target when attention is focused at the cued location and the target appears there. The distributed component reflects, in part, the amount of time taken to identify the target when it is presented at an uncued location. In Equation 3, $\mathrm{RT}_{+}$is the amount of time taken to respond on a valid trial when attention is focused at the cued location. In Equation 4, RT - is the amount of time taken to respond on an invalid trial when attention is focused at the cued location. Van der Heijden (1989) discusses how best to estimate $\mathrm{RT}_{+}$and $\mathrm{RT}_{-}$, and a more thorough treatment of his method is included in the Appendix. $\mathrm{RT}_{\mathrm{n}}$ is an index of the distributed component, and this can be estimated directly from the average RT on neutral trials. The assumption is that on neutral trials, only the distributed attentional component operates.

Given this mathematical model, it is possible to compute estimates of the costs and benefits in the 20-80 and 80-20 conditions, on the assumption that the probabilitymatching procedure operates perfectly in the 50-50 condition. The latter assumption appears to be questioned by the present data, because inspection of Table 5 reveals that the subjects appeared to show a cued-side bias in the 50-50 condition. However, in order to test the significance of this bias, a one-sample $t$ test compared the sample of cued-side bias values from the 50-50 condition $(M=0.66, S D=0.43)$ with the population mean of 0.5 . The test revealed that there was no statistically significant difference between the no-bias value of 0.5 and the sample mean of 0.66 in the $50-50$ condition $[t(11)=$ $1.24, p>.05$, one-tailed test]. On these grounds, therefore, it seems reasonable to apply the method described by van der Heijden (1989; the methods and estimated values are provided in Appendix A). In summary, the results of this exercise showed that the two-process model overestimates the size of the cuing effects in the 80-20 condition, and that it underestimates the size of the cuing effects in the 20-80 condition. Therefore, the present data deviate systematically from the quantitative predictions derived from the two-process model, and do not fit comfortably with any simple ideas about overall probability matching.

\section{EXPERIMENT 4}

To examine the nature of auditory orienting in further detail, performance was assessed with spoken cues. Previously, the cues were pure tones presented at the location of the target, and were analogous to the peripheral cues used in visual cuing experiments. Typically, in vi- 
sual cuing experiments, the subjects respond to the onset of the brightening of a box presented in peripheral vision. When a central cue is used in such studies, it is a suitably oriented arrowhead symbol presented at the center of the display. In this experiment, the auditory analogue of the central cue consisted of one of the spoken words "left," "right," or "mid," presented binaurally, and therefore heard in a central position. On trials with a valid cue, the word "left" or "right" described the location of the target, whereas on trials with an invalid cue, the word "left" or "right" described the location contralateral to the location of the target; "mid" constituted the neutral cue (cf. Murray et al., 1988). Here again, subjects responded with fingers of the same hand, as described in Experiment 3.

The early distinction between automatic (exogenous) and controlled (endogenous) orienting components has been developed and elaborated for the visual modality by Müller and coworkers (Müller \& Findlay, 1988; Müller \& Rabbitt, 1989; for a related idea, see also Weichselgartner \& Sperling, 1987). According to Müller, peripheral cues trigger distinct attentionalprocessing components. The automatic component is fast acting (i.e., it fades out within 100-300 msec of the cue onset), and it is reflexive (i.e., it cannot be interrupted). In contrast, the controlled component has a longer rise time, is less effective, and shows longer persistence. In addition, whereas the automatic component is little affected by cue validity, the controlled component leads to spatial expectancy on the basis of the probability of the cue type.

In a detailed examination of these ideas, Müller and Findlay (1988) compared visual cuing with peripheral and central cues, across a range of SOAs. In very general terms, the data showed that, with peripheral cues, benefits were present for SOAs as short as $50 \mathrm{msec}$, showing a slight increase up to an SOA of $125 \mathrm{msec}$, and then declining for SOAs up to $500 \mathrm{msec}$. Costs were present early on, but continued to decline as the SOA increased. With central cues, however, the pattern was different. Although very short SOAs were not examined, costs and benefits were found at an SOA of $100 \mathrm{msec}$. Broadly, the size of the cuing effects increased between 100 and $150 \mathrm{msec}$ and then decreased as the SOA increased further.

By strict analogy, this pattern of results suggests that when a central cue is used in the auditory domain, the size of the cuing effects should tend to decrease over the range of SOAs tested. This is based on the assumptions that the central cue invokes controlled processes, and that controlled processes in audition act in a similar way to those described in the visual modality. To test these ideas, Experiment 4 examined performance over a range of SOAs using central cues that were informative about the target's location. As a confirmation that cuing effects could be demonstrated for these subjects, they were also run in a single block of trials with the same peripheral cuing procedure that was used in Experiment 3.
A practical constraint was imposed on the experiment by the overall duration of the spoken word cues. The minimum duration of word tokens that were both accurately recognized and natural sounding was about $280 \mathrm{msec}$, so all the cues were edited to be of this length. This meant that at a $0-\mathrm{msec}$ ISI, the SOA was $280 \mathrm{msec}$. As a consequence, the experiment does not shed light on any possible early cuing effects that might arise at SOAs shorter than $280 \mathrm{msec}$. Thus the present results do not provide information about any automatic central auditory cuing effects that may arise with very short SOAs.

\section{Method}

\section{Subjects}

Twelve undergraduates from the University of York acted as subjects. Some were given course credit for participating, while others were paid $\mathfrak{f 3}$. As before, they were selected without regard to gender, and none reported a hearing impairment.

\section{Design and Procedure}

Tokens of the three cue words "left," "right," and "mid" were recorded in a male voice in a sound-attenuating room. The words were low-pass filtered $(3.5-\mathrm{kHz}$ corner frequency) and digitized with a sampling rate of $10 \mathrm{kHz}$. Tokens of each word, selected to be of roughly equal duration, were edited digitally to be exactly $280 \mathrm{msec}$ long, and their levels were normalized so as to have equal total energy. The cue words were always presented binaurally, and were thus perceived in a central location. For the peripheral cuing condition, a $2-\mathrm{kHz}$ cue tone was created with duration and level equal to that of the words, and linear onset and offset amplitude ramps equal to $5 \%$ of the total duration. The cue tone was presented either to the left ear, to the right ear, or binaurally; when presented binaurally, the level of the cue tone presented to each ear was decreased by $3 \mathrm{~dB}$. For both central and peripheral cuing conditions, the target was always a $400-\mathrm{Hz}$ tone of $100-$ msec duration, and was presented to the left and the right ears with equal probability, as in the previous experiments.

In the central-cue condition, six values of the interval between cue offset and target onset were used. These translated into SOAs of $280 \mathrm{msec}$ (here, the target followed the cue immediately), 330, $380,480,680$, and $1,080 \mathrm{msec}$. For each SOA, there were 30 practice trials and 120 experimental trials. 40 of the experimental trials were neutral (the cue word "mid" was presented), and of the remaining 80 trials, $64(80 \%)$ were valid (i.e., the cue word was "right" when the target was on the right, and "left" when it was on the left) and $16(20 \%)$ were invalid (i.e., the cue word was "right" when the target was on the left, and vice versa). Six orders of presentation of the SOA conditions were constructed, so that across the six orders, each condition was preceded by every other condition once. Two subjects were assigned randomly to each order.

Each subject also served in the brief peripheral cuing condition with 280-msec SOA, in which the proportion of valid, invalid, and neutral trial types was the same as above. Half of the subjects were given the peripheral cuing condition first (before the central cue conditions), and the other half were given it last (after the central cue conditions).

In all other respects, the experimental procedure was the same as that described previously. The subjects were not given any information about the probability of the different cue types.

\section{Results}

Summary statistics of the mean RTs for the conditions of interest are shown in Table 6. All RTs that were less than $100 \mathrm{msec}$ or further than $\pm 3 S D$ s away from 
each subject's condition mean were discarded prior to analysis of the RTs. Separate analyses were carried out on the RT data and the error data. Summaries of the error data are included in Table 7. As in the previous experiments, only incorrect responses were analyzed.

\section{Analysis of the Data for the Central Cuing Trials}

RTs. In this case, the data were entered into a $6 \times 3$ repeated measures ANOVA, with SOA $(280,330,380$, 480,680 , or $1,080 \mathrm{msec}$ ) and cue type (valid, invalid, or neutral) as fixed factors and subjects as a random factor. This analysis revealed that whereas the main effect of cue type reached statistical significance $[F(2,22)=$ $10.67, p<.001]$, neither the main effect of SOA nor the SOA $\times$ cue-type interaction was statistically reliable $(F<1.0$ for both). Post hoc analysis of the statistically significant main effect of cue type revealed that RTs were fastest in the valid condition and slowest in the invalid condition $(p<.05$, Tukey HSD test, for the pairwise comparisons relative to the responses on the neutral trials).

Analysis of the benefits and costs for the RTs produced no significant effects $[F<1.0$, for the main effects of SOA and cuing effects, and $F(5,55)=1.13, p>.05$, for the SOA $\times$ cuing-effects interaction]. Thus the pattern of results shows that there were statistically reliable benefits and costs, that these effects did not differ in size, and that they were unaltered across the range of SOAs tested.

Errors. Analysis of the error scores revealed that the main effects of SOA $[F(5,55)=3.33, p<.05]$ and cue type $[F(2,22)=49.66, p<.001]$ reached statistical significance. The interaction of these two factors was not, however, statistically reliable $[F(10,110)=1.09, p>$ $.05]$. A post hoc analysis of the main effect of cue type revealed that overall, the subjects made more errors on invalid trials than they did on either valid or neutral trials $(p<.01$, both comparisons Tukey HSD test). In turn, the number of errors made on valid and neutral trials did not differ $(p>.05$, Tukey HSD test). Overall,

Table 6

Mean Reaction Times (in Milliseconds) for Experiment 4

\begin{tabular}{|c|c|c|c|c|c|}
\hline \multirow{2}{*}{$\begin{array}{c}\text { Stimulus Onset } \\
\text { Asynchrony } \\
\text { (msec) }\end{array}$} & \multicolumn{3}{|c|}{ Cue Type } & \multicolumn{2}{|c|}{ Effect } \\
\hline & Valid & Neutral & Invalid & Benefit* & Cost $\dagger$ \\
\hline \multicolumn{6}{|c|}{ Central Cuing } \\
\hline 280 & 288 & 314 & 331 & 26 & 17 \\
\hline 330 & 294 & 318 & 331 & 24 & 13 \\
\hline 380 & 293 & 326 & 335 & 33 & 9 \\
\hline 480 & 310 & 326 & 335 & 16 & 9 \\
\hline 680 & 306 & 320 & 352 & 14 & 32 \\
\hline 1,080 & 302 & 317 & 342 & 15 & 25 \\
\hline \multicolumn{6}{|c|}{ Peripheral Cuing } \\
\hline 280 & 307 & 347 & 428 & 40 & 81 \\
\hline
\end{tabular}

*The benefits were computed by subtracting the valid scores from the neutral scores. The costs were computed by subtracting the neutral scores from the invalid scores.
Table 7

Mean Percentages of Error Scores* for Experiment 4

\begin{tabular}{ccccc}
\hline $\begin{array}{c}\text { Stimulus Onset } \\
\text { Asynchrony } \\
\text { (msec) }\end{array}$ & \multicolumn{3}{c}{ Cue Type } \\
\cline { 2 - 3 } & Valid & Neutral & Invalid & Cued-Side Bias $\dagger$ \\
\hline \multicolumn{5}{c}{ Central Cuing } \\
330 & 1.95 & 1.67 & 5.73 & 0.50 \\
380 & 1.56 & 0.63 & 4.69 & 0.34 \\
480 & 1.69 & 1.04 & 4.69 & 0.47 \\
680 & 1.43 & 1.67 & 1.56 & 0.19 \\
1,080 & 0.39 & 0.63 & 1.56 & 0.25 \\
\multicolumn{5}{c}{ Peripheral Cuing } \\
280 & 0.65 & 0.83 & 1.56 & 0.19 \\
& 1.04 & 1.88 & 7.80 & 0.47 \\
\hline
\end{tabular}

*These values were computed from just the incorrect responses and do not include the outliers discarded prior to the RT analysis. 'The derivation of the cued-side-bias values is given in the text.

$1.28 \%$ errors were committed on valid trials, compared with $1.08 \%$ errors on neutral trials.

Analysis of the benefits and costs in the accuracy data revealed that only the main effect of cuing effects reached statistical significance $[F(1,11)=30.49, p<$ $.001]$. Both the main effect of SOA $(F<1.0)$ and the SOA $\times$ cuing-effects interaction $[F(5,55)=2.22, p>$ $.05]$ failed to reach statistical significance. The statistically significant main effect of cuing effects was consistent with the previous analysis in showing that overall, and relative to the neutral baseline, more errors were made on invalid trials than were made on valid trials; thus the data revealed significant costs in the absence of significant benefits.

Estimates of cued-side bias were computed as described above, and their arcsin-transformed counterparts were analyzed in a one-way repeated measures ANOVA. The analysis showed that the main effect of SOA failed to reach statistical significance $[F(5,55)=1.03, p>$ $.05]$. In this case, none of the computed values exceeded 0.5 , showing that there was a slight tendency to press the key contralateral to that designated by the spoken word cue. Such a response bias cannot explain the presence of the significant RT benefits witnessed on valid trials. The analysis showed that the response bias did not vary as a function of the SOA.

\section{Analysis of the Data for the Peripheral Cuing Trials}

RTs. RT scores were also analyzed for the peripheral cuing trials using a one-way repeated measures ANOVA, which revealed a statistically significant effect of cue type $[F(2,22)=40.75, p<.0001]$. The benefits and costs were analyzed using a paired $t$ test, which showed that the size of the costs was significantly greater than that of the benefits $[t(11)=3.56, p<.01$, two-tailed test].

Errors. Analysis of the corresponding error scores also revealed a statistically significant effect of cue type $[F(2,22)=11.86, p<.001]$. The benefits and costs as- 
sociated with the errors were analyzed using a paired $t$ test. Although the benefits were numerically smaller than the costs, this difference just failed to reach statistical significance $[t(11)=-2.04, p>.05]$.

\section{Discussion}

This experiment has shown significant costs and benefits at all tested SOAs. In the RT data, the costs and benefits were symmetrical about the neutral baseline. In the error data, although the effects were very small, the costs were greater than the benefits. The size of the cuing effects remained unchanged as the SOA increased from $280 \mathrm{msec}$ to $1,080 \mathrm{msec}$. This result contrasts with those from studies in the visual cuing literature (Müller \& Findlay, 1988), which have shown a tendency for the size of the cuing effects to decrease as the SOA increased. Nevertheless, the general pattern of performance is consistent with the idea that the central cue invokes a controlled (endogenous) component of directed attention by allowing "active maintenance of orienting to the cued position" coupled with an inhibitory effect for the less likely location (Müller \& Findlay, 1988, p. 152). In the auditory domain, though, the insensitivity of the cuing effects to variation in the SOA as it increased up to $1,080 \mathrm{msec}$ suggests that subjects can maintain their focus of attention on the cued location for a longer duration than they can in the visual domain. Overall, the present results from the central cuing paradigm are consistent with the notion of controlled orienting in the auditory domain, discussed earlier in relation to peripheral auditory cuing (see Spence \& Driver, 1994).

The response-bias data are of particular note. Most importantly, the present cuing effects arose when subjects showed a tendency to respond to the side opposite to that named by the cue. In this respect, there is a clear dissociation between the attentional cuing effects and the response-bias effects. This particular pattern of data provides further strong evidence of an auditory orienting component separate from any later response components. A supplementary point in this regard is that the presence of the response-bias effects militates against any simple probability-matching account of the data. Such an account would posit a tendency to respond to the location named by the cue because the cue in some way "signaled" the most probable location of the target; however, no such tendency was evident in our data.

The peripheral cuing control condition in this experiment allows a direct comparison to be made between performance with central cues and performance with peripheral cues. To this end, the RT data from the peripheral cuing condition were analyzed, together with those from the $280-\mathrm{msec}$ SOA central cuing condition. The SOA was identical across these two conditions. Here, the overall size of the cuing effects (i.e., costs + benefits) was computed for each subject in each of the two conditions. Given that there has been some controversy over how best to deal with performance on neutral trials (Jonides \& Mack, 1984), it was thought inappro- priate to treat the different sorts of neutral trials in the central and peripheral cuing trials as being equivalent. Therefore, the magnitude of the difference in RTs between invalid and valid trials was examined. The corresponding data were analyzed in a paired $t$ test, which revealed that the mean cuing effect in the central condition (43 msec) was statistically smaller than that found in the peripheral cuing condition $(121 \mathrm{msec})[t(11)=-3.45$, $p<.01$, one-tailed test]. This difference is as predicted on the basis of the similar findings in the visual domain (Müller \& Findlay, 1988, Experiment 4). It fits well with the notion that when cue validity is less than $100 \%$, the automatic orienting effect of a peripheral cue adds to "voluntary" preparation processes. Central cues, in contrast, cannot be discriminated on the basis of their presentation location, and so lack the crucial spatial property of a peripheral cue. Therefore, larger cuing effects arise with peripheral cues than with central cues (see Müller \& Rabbitt, 1989, for a more thorough discussion of this point).

One further point of interest relates to performance in the peripheral cuing condition. In this experiment, the data showed that the size of the costs was significantly greater than the size of the benefits. This pattern contrasts with performance in Experiments 2 and 3, in which peripheral cues gave rise to costs and benefits of equivalent size. This contrast may be attributable to differences in the SOAs. In Experiments 2 and 3, the SOA was set at $100 \mathrm{msec}$, whereas in Experiment 4, it was $280 \mathrm{msec}$, or nearly three times that used previously. The pattern found in Experiment 4 seems to fit with the idea that, at longer SOAs, performance, even with peripheral cues, reflects the operation of a controlled orienting component. To adopt arguments put forward in the visual cuing literature (see Müller \& Findlay, 1988), such a component may operate to inhibit the processing of the target at the uncued location. However, until auditory peripheral cuing is systematically examined across a range of SOAs, the nature of the underlying mechanisms remains unknown.

\section{GENERAL DISCUSSION}

In the present study, and across a range of different tasks, further evidence for the existence of auditory orienting has been uncovered. In Experiment 1, a small but statistically reliable difference showed that subjects tended to respond more quickly when cue and target were presented to the same ear than when the stimuli were presented to different ears. Here, the stimuli were delivered over headphones and the subjects responded to the onset of target tone. Given that the size of the effect was small, further examination of simple tone detection was abandoned, and the remaining experiments used choice-RT paradigms. Previously, Rhodes (1987) has argued that evidence for auditory cuing is elusive because target detection may be performed on the basis of information registered before the specification of auditory lo- 
cale is available. According to Rhodes, cuing effects concerning stimulus location might, however, be found when tapping "higher level auditory representations" $(1987$, p. 2) that code spatial information. The data from Experiment 1 suggest that, in contrast to Rhodes's proposal, an attentional component may be operating at a very peripheral stage of auditory processing.

Further evidence for auditory orienting comes from the next two experiments. In Experiment 2, subjects responded with a keypress on the side of the target's location. Under these conditions, significant costs and benefits arose and, moreover, these effects were modulated by the probability with which a cue type occurred. The smallest effects were observed when valid cues were rare relative to invalid cues. The data also suggested that subjects exhibited a response bias to respond to the location of the cue. Nevertheless, formal estimates of response bias suggested that there was no evidence of a systematic change in bias across the different cue-typeprobability conditions. It is unlikely, therefore, that a systematic change in bias caused the systematic change in cuing effects witnessed in the RT data.

In Experiment 3, the subjects again made choice RTs, but with a different response arrangement. In this case, they responded with fingers on the same hand, and the response box was aligned so as to be perpendicular to their body midline. This reduction in stimulus-response compatibility sought to minimize any cuing effects that were due purely to response artefacts. The results again showed that significant cuing effects arose for all cue probabilities. As before, the size of the cuing effects varied as a function of the probability of the different cue types: cuing effects were larger when valid cues were frequent, in comparison with conditions in which valid cues were rare.

The pattern of results found in Experiments 2 and 3 are generally in good agreement, and two particular findings deserve further consideration. First, the magnitudes of the cuing effects for informative and uninformative cues were equivalent, and second, although significant cuing effects were found when the valid cue occurred infrequently, these were diminished relative to the other two conditions. All of the observed cuing effects were obtained with an SOA of $100 \mathrm{msec}$, which is treated in the visual cuing literature as a short duration. Overall, this pattern of performance fails to conform to the account of attentional processes set out by Posner and Snyder (1978). Nevertheless, the data can be accommodated within a general framework that posits separate automatic and controlled components, but only if a less strict reading of the term automatic is adopted. Certain characteristics of the attentional orienting examined here do match those associated with traditional notions of automatic processing. Those characteristics are that it was invoked by peripheral cues, at short SOAs, when subjects were unaware of the cue-type probability, and that processing was enhanced for a cued location relative to an uncued location for all cue-type probabil- ities. However, it appears that the present cuing effects are neither unmodifiable nor resistant to subjective control. In both Experiment 2 and Experiment 3, it was found that the cuing effects were modulated by the cuetype probability. Even though the cuing effects were equivalent for informative and uninformative cues, when the valid cue occurred infrequently, the size of the cuing effects diminished accordingly. Such a modulation in the effects should not have arisen if the orienting were purely automatic, in its traditional definition.

The modulation of cuing effects demonstrated in our experiments augments Spence and Driver's (1994) finding that automatic orienting in the auditory domain can be overridden by higher-order strategic considerations. The present data show that it is not essential to inform subjects explicitly about the probability of different cue types; simply altering the cue-type probability can modulate auditory orienting. It is clear that further studies are needed to explore more thoroughly the present suggestion that auditory orienting exhibits a considerable degree of subjective control. Nevertheless, at present, it seems that even though a peripheral cue can induce orienting automatically in the normal course of events, such a tendency can be modified by both environmental contingencies and internal control.

Spence and Driver (1994) choose to hold on to the distinction between exogenous and endogenous orienting mechanisms, arguing that the exogenous (automatic) component may be used as the default "when the subject has no particular intentions with regard to the stimulus" (p. 571). They agree that the exogenous component may be strategically modulated, but emphasize that the central issue is about the degree to which such modulation takes place, the suggestion being that some forms of orienting are more automatic than others. An alternative proposal, though, is espoused by Folk and coworkers (Folk, Remington, \& Johnston, 1992, 1993; but see Yantis, 1993), and is encapsulated in the contingent involuntary orienting hypothesis, which states that "all involuntary shifts of attention are ultimately contingent on variable internal control settings" (Folk et al., 1993, p. 682). The general idea here is that the subject, in some sense, fixes an attentional control setting so as to respond selectively to the current task-relevant stimulus properties. Once this system is set, however, orienting is then purely stimulus driven. The fundamental claim is that so-called involuntary shifts of attention are ultimately governed by the internal control setting. It therefore makes no sense to draw up a dichotomy between controlled and automatic orienting, because orienting in general is determined in relation to the internal control setting. Although the present data in no way resolve the general issue of attempting to define type-distinct orienting mechanisms, they fit rather uneasily with the traditional controlled/automatic orienting distinction in the ways discussed above. Further experiments need to explore whether or not the contingent involuntary orienting hypothesis provides a useful framework for thinking about 
attentional control in the auditory domain. Indeed, it would seem appropriate in future work to examine performance when subjects are informed explicitly about the experimental cue-target contingencies.

The present data also provide some further constraints for the development of quantitative models of selective attention. The data analysis suggests that a strictly linear probability-matching procedure cannot account for performance. A possible alternative is to conceive of a peripheral cue accruing processing resources up to a threshold level that cannot be exceeded. Such a claim appears warranted because the effects of informative and uninformative cues were similar in terms of size. However, when the cue predominantly signals another target location (as in the $20-80$ conditions used here), fewer resources may be dedicated to the cue's own location.

In the final experiment, informative central cues were used and a range of SOAs was tested. Under these conditions, significant cuing effects were again found. The size of the cuing effects was constant over the range of SOAs tested, the same amount of cuing being found at $280-\mathrm{msec} \mathrm{SOA}$ as at 1,080 -msec SOA. Importantly, these effects arose when subjects showed a slight bias to respond to the side that was contralateral to that of the target. This pattern of results shows a clear dissociation between attentional and response component processes and provides firm evidence for central auditory orienting.

Experiment 4 also showed that central cuing effects were reliably smaller than the corresponding peripheral cuing effects at short SOAs $(280 \mathrm{msec})$, a result that matches that found in the visual domain, where, at SOAs of less than $400 \mathrm{msec}$, central cuing effects are smaller than peripheral cuing effects. Müller and Findlay (1988) explained their results in terms of a peripheral cue invoking an automatic component that both facilitates processing at the cued location and inhibits processing at uncued locations. They suggested that both of these effects dissipate with time and may be compensated for by the operation of a controlled component. In extending these ideas, though, Müller and Rabbitt (1989) suggested that the overall difference in the size of the two kinds of cuing effects is due to the peripheral cue invoking both voluntary and automatic processes, whereas the central cue invokes just voluntary processes. According to this view, when cue validity is less than $100 \%$, the automatic orienting effect of a peripheral cue adds to voluntary preparation processes, to produce a comparatively larger cuing effect than that produced by a central cue.

In conclusion, auditory orienting has been demonstrated with peripheral cues using simple RTs, and with peripheral and central cues using choice RTs. In all cases in which choice RTs have been measured, the effects appeared to be sensitive to attentional control. With peripheral cues, the cuing effects were modulated by the probability of the cue type. With central (informative) cues, the size of the cuing effects appeared not to vary as the interval between the cue and the target increased. Al- though in the visual cuing literature, the distinction between automatic and controlled processes has been used successfully to help provide accounts of performance, the present work is in keeping with more recent concerns about the degree to which automatic orienting is insulated from strategic control. It is possible that only automatic processes are involved during simple tone detection, but the general picture is that more complex auditory processes demand attentional resources.

\section{REFERENCES}

Buchtel, H. A., \& ButTer, C. M. (1988). Spatial attentional shifts: Implications for the role of polysensory mechanisms. Neuropsychologia, 26, 499-509.

Butter, C. M., Buchtel, H. A., \& SAntucci, R. (1989). Spatial attentional shifts: Further evidence for the role of polysensory mechanisms using visual and tactile stimuli. Neuropsychologia, 27, 1231. 1240.

DunCan, J. (1980). The demonstration of capacity limitation. Cognitive Psychology, 12, 75-96.

ERIKSEN, C. W., \& YEH, Y.-Y. (1985). Allocation of attention in the visual field. Journal of Experimental Psychology: Human Perception \& Performance, 11, 583-597.

FolK, C. L., Remington, R. W., \& Johnston, J. C. (1992). Involuntary covert orienting is contingent on attentional control settings. Journal of Experimental Psychology: Human Perception \& Performance, 18, 1030-1044.

Folk, C. L., Remington, R. W., \& Johnston, J. C. (1993). Contingent attentional capture: A reply to Yantis (1993). Journal of Experimental Psychology: Human Perception \& Performance, 19, 682685.

JoNiDEs, J. (1980). Towards a model of the mind's eye's movement. Canadian Journal of Psychology, 34, 103-112.

JoNIDES, J., \& MACK, R. (1984). On the cost and benefits of cost and benefits. Psychological Bulletin, 96, 29-44.

KeELE, S. W. (1973). Attention and human performance. Pacific Palisades, CA: Goodyear.

Klein, R., \& Hansen, E. (1990). Chronometric analysis of apparent spotlight failure in endogenous visual orienting. Journal of Experimental Psychology: Human Perception \& Performance, 16, 790-801.

Lowe, G. (1968). Auditory detection and recognition in a twoalternative, directional uncertainty situation. Perception \& Psy. chophysics, 4, 180-182.

MAYLOR, E. A. (1985). Facilitatory and inhibitory components of orienting in visual space. In M. I. Posner \& O. S. M. Marin (Eds.), Attention and performance $X I$ (pp. 189-204). Hillsdale, NJ: Erlbaum.

Mazzucchi, A., Cattelani, R., \& Umiltà, C. (1983). Hemispheric prevalence in acoustical attention. Brain \& Cognition, 2, 1-11.

MülLER, H. J. (1994). Qualitative difference in response bias from spatial cueing. Canadian Journal of Experimental Psychology, 48, 218-241.

Müller, H. J., \& FindLAY, J. M. (1988). The effect of visual attention on peripheral discrimination thresholds in single and multiple element displays. Acta Psychologica, 69, 129-155.

Müller, H. J,, \& RABbIT, P. M. A. (1989). Reflexive and voluntary orienting of visual attention: Time course of activation and resistance to interruption. Journal of Experimental Psychology: Human Perception \& Performance, 15, 315-330.

MURRay, J., Allard, F., \& Bryden, M. P. (1988). Expectancy effects: Cost-benefit analysis of monaurally and dichotically presented speech. Brain \& Language, 35, 105-118.

POSNER, M. I. (1978). Chronometric explorations of mind: The third Paul Fitts lectures. Hillsdale, NJ: Erlbaum.

Posner, M. I., Nissen, M. J., \& KLeIN, R. (1976). Visual dominance: An information-processing account of its origins and significance. Psychological Review, 83, 157-171.

Posner, M. I., NisSen, M. J., \& Ogden, W. C. (1978). Attended and un- 
attended processing modes: The role of set for spatial location. In H. L. Pick, Jr., \& E. Saltzman (Eds.), Modes of perceiving and processing information (pp. 137-157). Hillsdale, NJ: Erlbaum.

POSNER, M. I., \& SNYDER, C. R. (1978). Facilitation and inhibition in the processing of signals. In P. M. A. Rabbit \& S. Dornic (Eds.), Attention and performance $V$ (pp. 669-682). London: Academic Press.

RHODEs, G. (1987). Auditory attention and the representation of spatial information. Perception \& Psychophysics, 42, 1-14.

SCHARF, B. (1988). The role of listening in the measurement of hearing. Advances in audiology (Vol. 5, pp.13-26). Basel, Switzerland: S. Karger.

Simon, J. R., ACosta, E., JR., \& Mewaldt, S. P. (1975). Effect of locus of warning tone on auditory choice reaction time. Memory \& Cognition, 3, 167-170.

SPENCE, C. J., \& Driver, J. (1994). Covert spatial orienting in audition: Exogenous and endogenous mechanisms. Journal of Experimental Psychology: Human Perception \& Performance, 20, 555-574.

van Der HeIJden, A. H. C. (1989). Probability matching in visual selective attention. Canadian Journal of Psychology, 43, 45-52.

Weichselgartner, E., \& Sperling, G. (1987). Dynamics of automatic and controlled visual attention. Science, 238, 778-780.

WINER, B. J. (1962). Statistical principles in experimental design. New York: McGraw-Hill.

YANTIS, S. (1993). Stimulus-driven attentional capture and attentional control settings. Journal of Experimental Psychology: Human Perception \& Performance, 19, 676-681.

\section{NOTES}

1. Spence and Driver (1994) independently decided on a similar manipulation in their examination of auditory orienting. The results of their experiments are discussed elsewhere in this paper.

2. There is no necessary implication that the focused/distributed distinction maps onto the previously defined automatic/controlled distinction, although van der Heijden (1989) seems to adopt such a mapping.

\section{APPENDIX}

Equations 3 and 4 are represented again here, as Equations $i$ and $\mathrm{ii}$, respectively:

$$
\mathrm{RT}_{\text {valid }}=p_{\text {cue }}\left(\mathrm{RT}_{+}\right)+\left(1-p_{\text {cue }}\right) \mathrm{RT}_{\mathrm{n}},
$$

and

$$
\mathrm{RT}_{\text {invalid }}=p_{\text {cue }}\left(\mathrm{RT}_{-}\right)+\left(1-p_{\text {cue }}\right) \mathrm{RT}_{\mathrm{n}},
$$

where $\mathrm{RT}_{\text {valid }}$ is the mean $\mathrm{RT}$ on valid trials, $\mathrm{RT}_{\text {invalid }}$ is the mean RT on invalid trials, and $p_{\text {cue }}$ is the probability of a valid cue. $\mathrm{RT}_{+}$is the amount of time taken to respond on a valid trial when attention is focused at the cued location, and RT is the amount of time taken to respond on an invalid trial when attention is focused at the cued location.

Using these equations, it is possible to compute estimated costs and benefits in the 20-80 and 80-20 conditions, if it is assumed that subjects are successfully probability matching in the 50-50 condition (after van der Heijden, 1989). Consider the computations for Experiment 3. Initially, the aim was to arrive at estimates for $\mathrm{RT}_{+}$and $\mathrm{RT}_{-}$. Here, $\mathrm{RT}_{\mathrm{n}}$ was taken as being the overall mean RT across all of the neutral trials in Experiment 3 (i.e., $443 \mathrm{msec}$ ). To estimate $\mathrm{RT}_{+}$from Equation $\mathrm{i}, p_{\text {cue }}$ was entered as $0.5, \mathrm{RT}_{\mathrm{n}}$ was entered as $443 \mathrm{msec}$, and $\mathrm{RT}_{\text {valid }}$ was simply the mean RT for valid trials taken from Table 4 (i.e., $387 \mathrm{msec}$ ). RT + works out to be $331 \mathrm{msec}$. By a similar method, and by entering appropriate values into Equation ii, RT _ was calculated to be $521 \mathrm{msec}$. The values thus calculated for $\mathrm{RT}_{+}$and RT - were then used to derive estimates for RTs on valid and invalid trials for the $20-80$ and $80-20$ conditions.

For the 20-80 condition, the estimated mean RTs on valid and invalid trials were, respectively, 421 and $459 \mathrm{msec}$. For the 80-20 condition, the estimated mean RTs on valid and invalid trials were, respectively, 353 and $505 \mathrm{msec}$. Although the individual estimates may be compared with their counterparts shown in Table 4, it is possible to compare the overall size of the estimated and obtained cuing effects for the two conditions (i.e., the size of the difference between RTs to valid and to invalid trials; alternatively, the sum of costs and benefits). The estimated cuing effect in the $20-80$ condition was $38 \mathrm{msec}$, and compares with the observed value of $49 \mathrm{msec}$. The estimated cuing effect in the 80-20 condition was $152 \mathrm{msec}$, and compares with the observed value of $116 \mathrm{msec}$. These comparisons reveal that the two-process model overestimates the size of the overall cuing effect in the 80-20 condition, and underestimates the size of the overall cuing effect in the 20-80 condition. As a consequence, the two-process model based upon probability matching does not provide a convincing account of performance in the present tasks. Moreover, the deviations from the model witnessed in the present data do not fit the pattern predicted on the basis of subjects moving from probability matching toward an optimal strategy (see van der Heijden, 1989, for more on this point).

Central to the present analysis is the assumption that subjects are successfully probability matching in the 50-50 condition. One indication of whether this is a reasonable assumption to make is whether or not subjects showed a cued-side bias in the 50-50 condition. Prior to the present analysis, a statistical test was carried out to see whether the sample of bias values deviated significantly from the no-bias value of 0.5 . This test failed to reveal such a statistically significant difference (see text for details). In turning to the results of Experiment 2, therefore, a similar preliminary test was used on the cued-sidebias values found in the $50-50$ condition. This test revealed that there was a statistically significant difference between the nobias value of 0.5 and the obtained bias values $[M=0.85, S D=$ $0.33 ; t(17)=4.45, p<.001$, one-tailed test]. Given this result, it would be inappropriate to apply the van der Heijden (1989) method to the results of Experiment 2.

(Manuscript received May 20, 1994; revision accepted for publication December 10, 1994.) 